\title{
Surgical management of pelvic Ewing's sarcoma in children and adolescents
}

\author{
HONGBIN FAN, ZHENG GUO, JUN FU, XIANGDONG LI, JING LI and ZHEN WANG \\ Department of Orthopedic Surgery, Xi-Jing Hospital, The Fourth Military Medical University, \\ Xi'an, Shaanxi 710032, P.R. China
}

Received June 16, 2016; Accepted March 9, 2017

DOI: $10.3892 / \mathrm{ol} .2017 .6677$

\begin{abstract}
The present study describes a novel surgical strategy used to treat immature pelvic Ewing's sarcoma (ES), one made possible owing to the intrinsic structure of the skeletally immature pelvis. A total of 12 children and adolescents with open triradiate cartilage received limb-salvage surgeries following a diagnosis of pelvic ES. In total, 3 patients with iliac lesions (2 lesions with extension into the sacrum) received surgical tumor excisions and allograft reconstructions. Another 8 patients with periacetabular lesions received trans-acetabular osteotomies and allograft reconstructions. No reconstruction was performed on 1 patient following excision of a pubic lesion. The median follow-up time was 39 months. At the time of the final follow-up appointment, 9 patients exhibited no evidence of disease, 2 patients had slow progressive pulmonary metastasis, 1 patient exhibited local recurrence of the disease and 1 patient had succumbed to the disease 24 months after surgery. Complications included issues with wound healing in 1 patient, a discrepancy in leg length in another and a screw loosening in a further patient. The patients had a mean Musculoskeletal Tumor Society score of 26 points and a mean International Society of Limb Salvage radiographic score of $90.1 \%$. The proposed surgical strategy for children and adolescents with pelvic ES in the present study could provide a feasible solution for acetabulum salvage and local tumor control.
\end{abstract}

\section{Introduction}

Ewing's sarcoma (ES) is the second most common malignant bone tumor in children and the fourth most common overall $(1,2)$. ES is most common during the second decade

Correspondence to: Dr Zhen Wang or Dr Zheng Guo, Department of Orthopedic Surgery, Xi-Jing Hospital, The Fourth Military Medical University, 15 West Changle Road, Xi'an, Shaanxi 710032, P.R. China

E-mail: wangzhen@fmmu.edu.cn

E-mail: guozheng@fmmu.edu.cn

Key words: pelvis, Ewing's sarcoma, children, adolescent of life, with the median age at diagnosis ranging from 13 to 19 years (3). Pelvic ES accounted for $19.9 \%$ of cases in the Mayo Clinic series (4) and $21 \%$ in the chapter written by Ginsberg et al (5), who discussed the principles and practice of ES. The numerous advances made in diagnostic imaging and multimodality therapy over the past few decades mean that the overall 5-year survival rate of ES has increased from $10 \%$ in the 1970 s (6) to $55-75 \%$ at the turn of the century (7). Studies have demonstrated that 5-year survival is improved in patients treated with surgical resection and chemotherapy vs. patients treated with chemotherapy and radiation or with radiation alone $(8,9)$.

However, patients with pelvic ES have a poorer prognosis compared with those that have lesions in their extremities. The 5-year survival rate for these patients is markedly lower (35\%) (8). Pelvic resections are classified by region according to studies by Enneking and Dunham (10), and O'Connor and Sim (11): Type I refers to resection of the ilium, type II to resection of the peri-acetabular region, type III to resection of the pubis or ischium and type IV to resection of the lateral mass of the sacrum. The resultant defects can cause substantial functional impairment. Various reconstructions, including fibular struts, hip transposition, arthrodesis and prosthetic replacements have been established. However, high rates of early and late complications have been reported $(12,13)$.

Unlike type I and type III resections, type II resections usually require excision of the entire acetabulum and lead to impaired hip function in adults. However, in skeletally immature children and adolescents, the pelvis has its own intrinsic structure. The triradiate cartilage closes at 16 to 18 years of age, thinning out prior to this $(14,15)$. This physis could act as a barrier to tumor extension, although it is not impenetrable. Prior to closure, it may represent an oncological margin for tumor resection, particularly for tumors that do not contact or partially contact the physis (16). As in metaphyseal tumors of long bones, in which an unaffected physis allows a transepiphyseal resection for joint-sparing, periacetabular tumors can be excised using trans-acetabular osteotomy based on triradiate cartilage. This can maximally preserve the unaffected acetabular components, which contribute to the growth of the acetabulum (15). Winkelmann (17) removed the sarcomas (types I and II) using a similar strategy. Sales de Gauzy et al (1) excised the periacetabular ES (types II and III) beyond the triradiate cartilage without reconstruction in 2 children. Nevertheless, there have 
been few reports in the literature documenting the surgical strategy for pelvic ES in children and adolescents, particularly for periacetabular lesions.

The present study retrospectively reviews the resection of pelvic ES and the following reconstructions in 12 children and adolescents in order to present intermediate-term results of using this surgical strategy as part of multimodality therapy.

\section{Patients and methods}

Patient demographics. Between January 2001 and October 2013, 12 children and adolescents with pelvic ES were treated surgically at the Department of Orthopedic Surgery, Xi-Jing Hospital (Xi'an, China). This series comprised 9 males and 3 females, with a mean age of 12.7 years (range, 7-16 years). Tumor sites were assigned according to the Enneking and Dunham classification (10). In total, 3 patients had type I (ilium) lesions, 2 of which extended into the sacrum (type I+IV); 8 had type II (periacetabular) lesions ( 3 above, 4 anterior inferior and 1 posterior inferior to the triradiate cartilage; termed type IIA, type IIB and type IIC, respectively, in this study); and 1 had a type III (ischiopubic) lesion (Fig. 1). Staging studies, including plain film, magnetic resonance imaging (MRI), chest computed tomography $(\mathrm{CT})$ and bone scintigraphy, were performed. All patients were non-metastatic at presentation.

Treatment protocol. All patients received vincristine ( $1.5 \mathrm{mg} / \mathrm{m}^{2}$ once a day), doxorubicin $\left(30 \mathrm{mg} / \mathrm{m}^{2}\right.$ every 2 days) and ifosfamide $\left(2,000 \mathrm{mg} / \mathrm{m}^{2}\right.$ every 3 days $)$ neoadjuvant chemotherapy for 12 weeks prior to surgery. No preoperative radiotherapy was performed. The response to chemotherapy was assessed by the Response Evaluation Criteria in Solid Tumors (RECIST) (18). Surgeries were performed between weeks 8 and 10 after the initiation of chemotherapy. Thereafter, chemotherapy was continued in all patients. Postoperative radiotherapy was administered to patients who received excisions with marginal margins (i.e., a margin through tissue that has gross or histological evidence of reaction to the tumor) or contaminated margins. Patients treated with Intensity Modulated Radiation Therapy were prescribed a dose of $45 \mathrm{~Gy}$ in 15 fractions (3 Gy/day for 15 days).

Depending on the location and extent of the tumor, four types of excision were performed singly or in combination. Part of the ilium and the surrounding gluteal muscles (type I) were excised in 1 patient with iliac lesions. Another 2 patients with iliac lesions required excision of a lateral mass of the sacrum besides the ilium and gluteal muscles (type I+IV). The following excisions were performed on 8 periacetabular lesions: 3 patients underwent resection of the upper component of the acetabulum and neighboring ilium (type I+IIA); 4 patients required removal of the anterior inferior component of the acetabulum, as well as all or part of the pubis and ischium (type IIB+III); 1 patient had the whole of the inferior component of the acetabulum, with the pubis and ischium, resected (type IIB+IIC+III); and 1 patient had a pubic lesion and underwent partial excision of the pubis (type III) (Table I).

As aforementioned, a 'marginal margin' is a margin through tissue that has gross or histological evidence of a reaction to the tumor. If there is an intact fascial boundary between the tumor and surgical resection margin, the margin is defined as 'wide' (19). A marginal margin typically occurred in certain areas of the specimen where the tumor grew very close to the neurovascular or genitourinary structures. In the present study, surgical margins were classified as wide in 8 patients and as marginal in 4 . The excised tumor was examined for safe margin by an experienced pathology technician. Samples from different sides of the removed tissue (proximal, distal, lateral, medial, top and bottom) were embedded in Optimal Cutting Temperature Compound, frozen, sectioned into $10 \mu \mathrm{m}$ thick slices and then stained with hematoxylin and eosin for $8 \mathrm{~min}$ at room temperature. During surgery, 2 patients were observed to have contaminated margins (into the lesion) and required further excision to yield negative margins. After 2008, 5 patients received precise resection using a computer-assisted navigation system (CANS; Stryker Pacific, Ltd., Hong Kong, China). The images from CT, MRI and bone scintigraphy were integrated in CANS and a three-dimensional (3D) tumor model was generated. This model was used for preoperative planning and navigation-guided resection, as reported previously (20). Of the 5 patients undergoing CANS, 1 underwent a type I excision, 1 underwent a type I+IV excision, 1 underwent a type IIB+III excision, 1 underwent a type I+IIA excision and 1 underwent a type III excision (Table I).

The reconstructions were individualized on the basis of variables that included patient age, functional demands and tumor extension (Table I). The pedicle screw/rod/allograft composite reconstructions were performed in 3 patients after a type I or type I+IV excision (Figs. 2 and 3). Reconstructions with allografts and plates were performed in 8 patients with periacetabular lesions (Figs. 4-9). No reconstruction was performed in 1 patient, who underwent a partial pubic resection (type III). Selecting allografts that could closely match the excised bone was important for periacetabular reconstruction. A 3D virtual bone bank system has been developed in the Department of Orthopedic Surgery, Xi-Jing Hospital. Using this system, 3D tumor models and allografts were measured and compared to determine the most appropriate match. Owing to the limited availability of pediatric allografts, the majority of allografts in the bone bank were over-sized and required cutting or trimming to match the defect as closely as possible.

Antibiotic prophylaxis (cefazolin, $1 \mathrm{~g} /$ day) was usually administered intravenously for the first week following surgery. Prophylaxis could be administered for longer, depending on multiple factors, including time of drain retention, medical co-morbidities and potential wound healing problems. A total of 4 patients who had positive margins in final pathology reports received postoperative irradiation (Table I).

Patients were required to undergo non-weight-bearing ambulation for the first 6-8 weeks after surgery. Partial weight-bearing ambulation was required between weeks 9 and 12 , and full weight-bearing ambulation after 12 weeks. However, the full weight-bearing time depended on radiographic healing time, which varied between 3 and 5 months after surgery.

Follow-up and evaluation. The study was approved by the Institutional Review Board of Xi-Jing Hospital. Informed consent was obtained from all patients and procedures followed were in accordance with the ethical standards of 


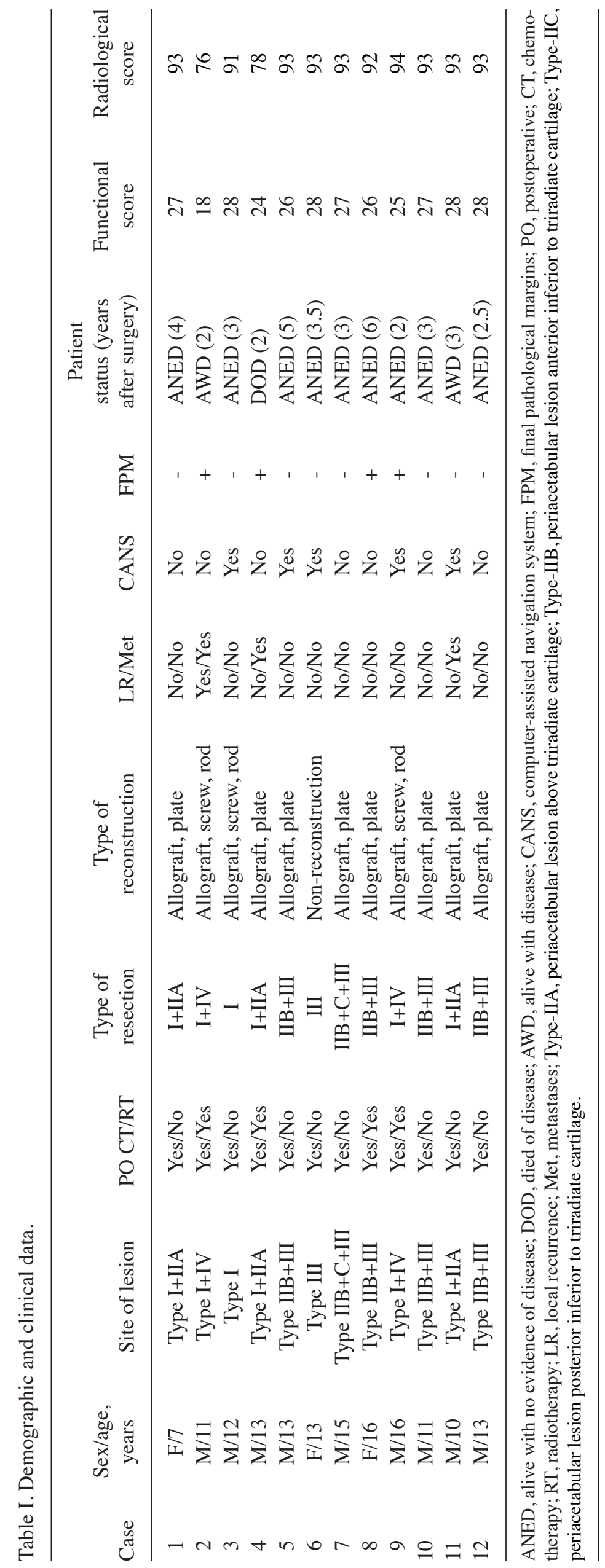



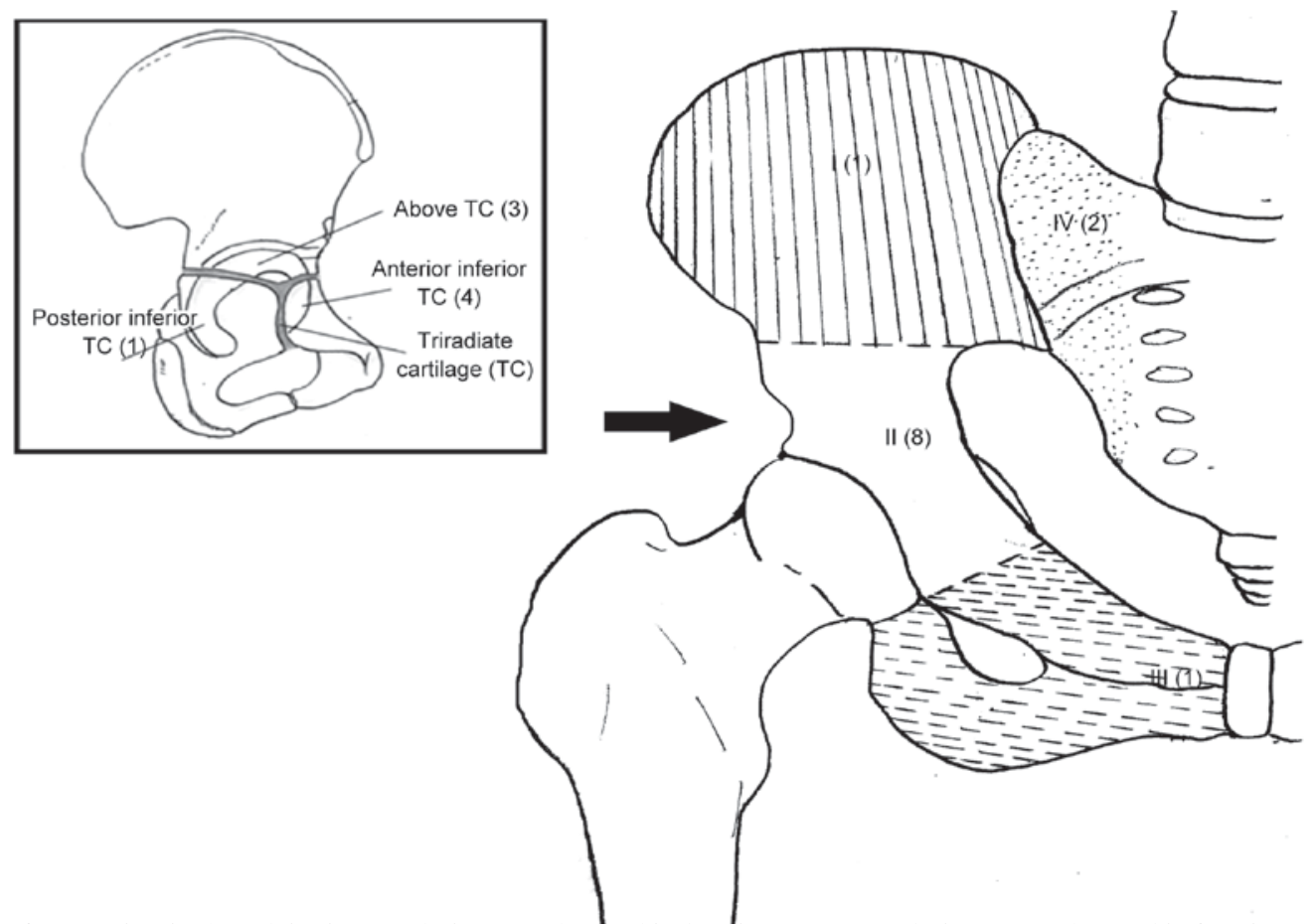

Figure 1. Schematic of tumor sites in the pelvis. A type I lesion was observed in 1 patient; type I+IV lesions were observed in 2 patients; type II lesions were observed in 8 patients ( 3 above, 4 anterior inferior and 1 posterior inferior to triradiate cartilage); and a type III lesion was observed in 1 patient.
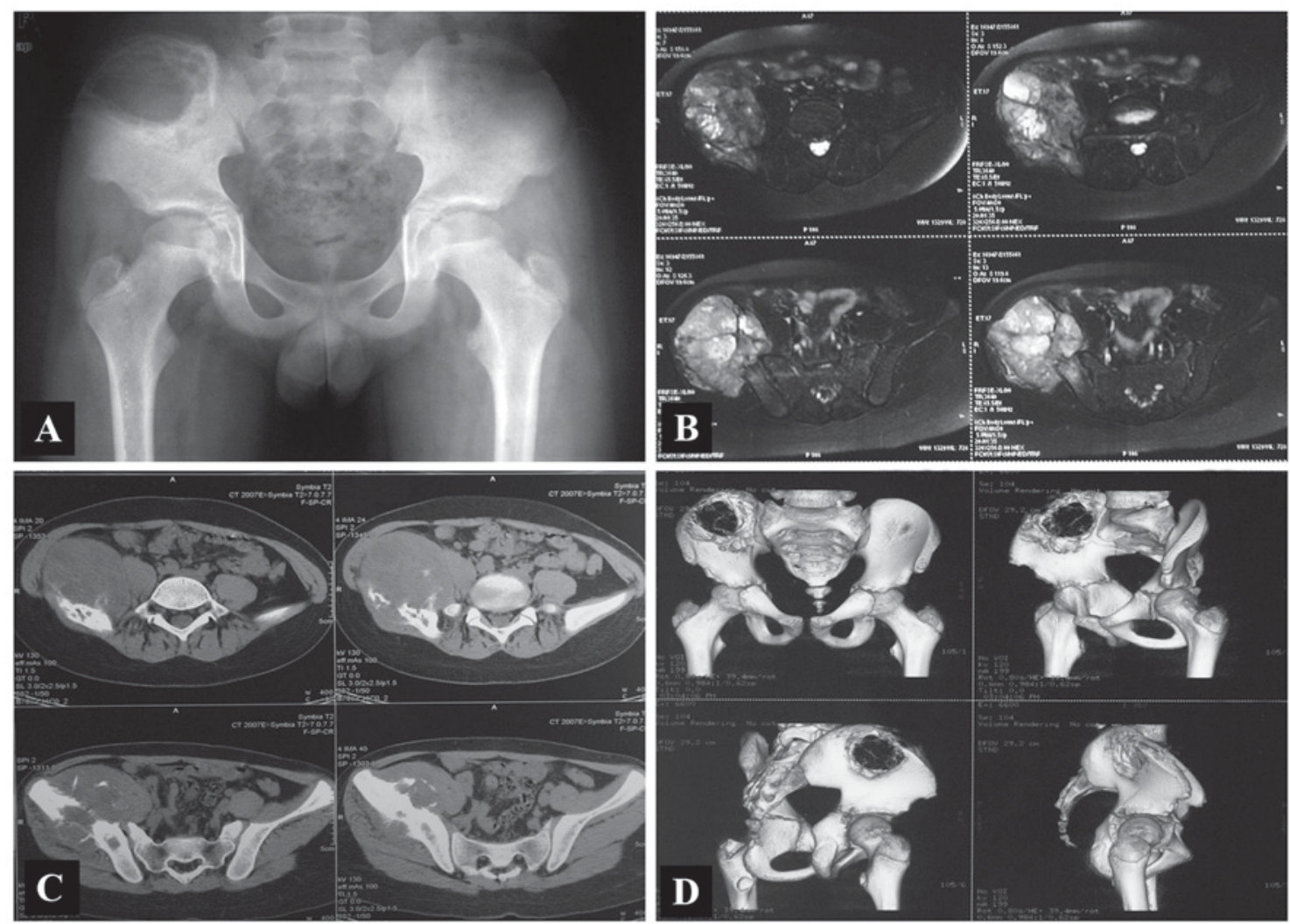

Figure 2. Type I lesion (case 3). (A) An X-ray showing a large osteolytic lesion in the right ilium. (B) The lesion exhibited high signal intensity on T2-weighted magnetic resonance imaging; (C) Computed tomography scan and (D) 3-dimensional reconstruction show the expandable osteolytic lesion with soft-tissue invasion.

the Responsible Committee on Human Experimentation of Xi-Jing Hospital and with The Helsinki Declaration. All patients completed the follow-up. The median follow-up time was 39 months (range, 24-72 months). Patients were assessed at 2 weeks, 1 month and 3 months after surgery, and every 3 months thereafter until 2 years after surgery, and then 
A

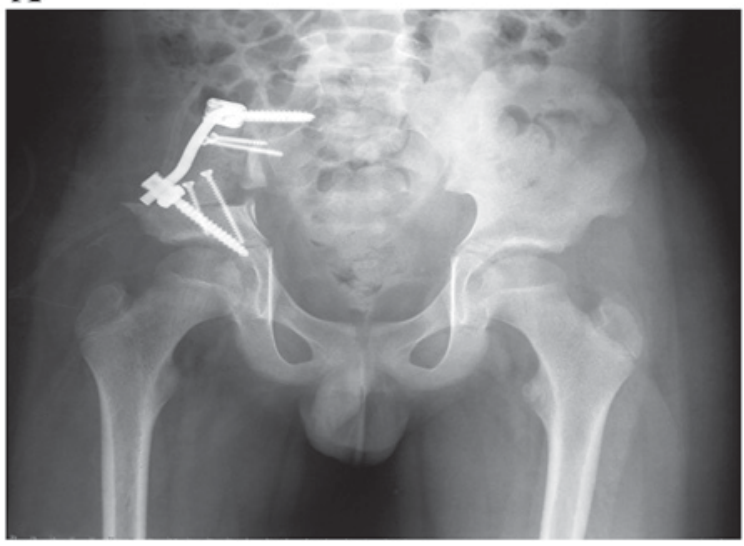

B

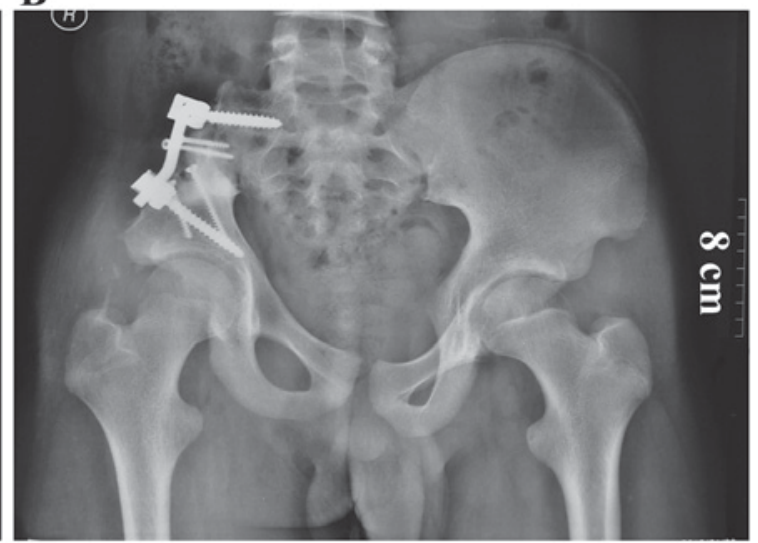

Figure 3. X-ray showing excision of the type I lesion and pedicle screw/rod/allograft reconstruction. Images taken (A) directly after the operation and (B) at 3 years after surgery.
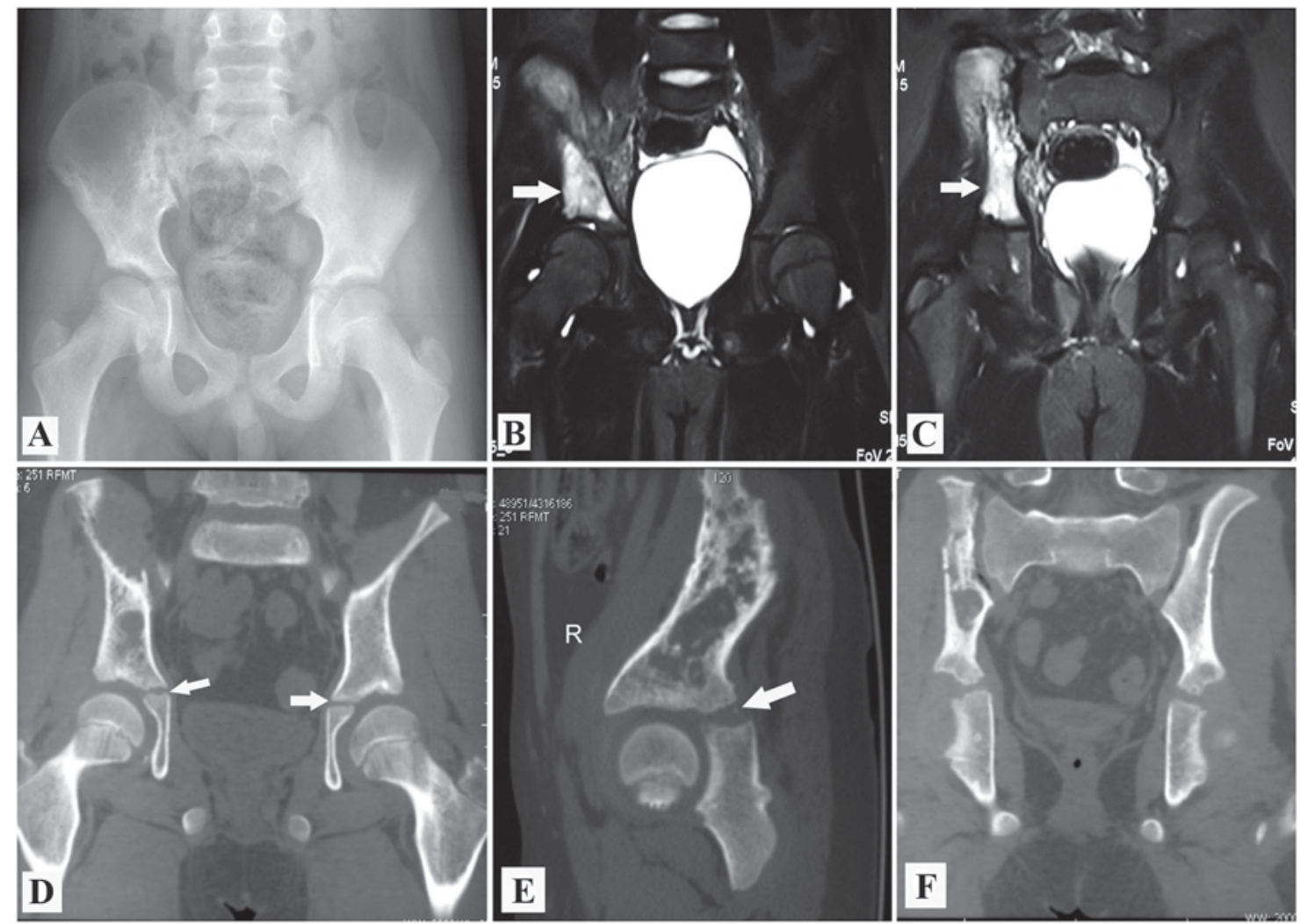

Figure 4. Type I+IIA lesion (case 11). (A) X-ray showing a large osteolytic lesion involving the upper component of the acetabulum and the neighboring ilium. (B and C) T2-weighted magnetic resonance imaging, showing high signal intensity indicative of the lesion (indicated by white arrow). (D-F) Images from a coronal and sagittal CT scan indicating that the triradiate cartilage was not penetrated by tumor (indicated by white arrow).

every 6 months. Plain radiographs and physical examinations were performed at each follow-up appointment. Chest CT was performed every 3 months until 2 years after surgery and then every 6 months to assess for metastatic disease. Functional outcomes were determined using the MSTS score 93 system (21). In brief, this system assigned numerical values $(0-5)$ for each of the 6 categories: Pain, function, emotional acceptance, support, walking and gait. The plain radiographs were evaluated according to the International Society of Limb Salvage (ISOLS) grading system (22), which had 5 categories, including healing of osteotomies (union), contour of the graft (graft shortening), graft fracture, density of the graft (resorption) and stability of implant. The numerical values (1-4) were assigned for each category to indicate poor, fair, good and excellent, respectively. The score was calculated by adding the value for each criterion and dividing by the maximum attainable score. This was then expressed as a percentage with a maximum score of $100 \%$. The MSTS and ISOLS scores were obtained and reported at the time of the final follow-up appointment. 


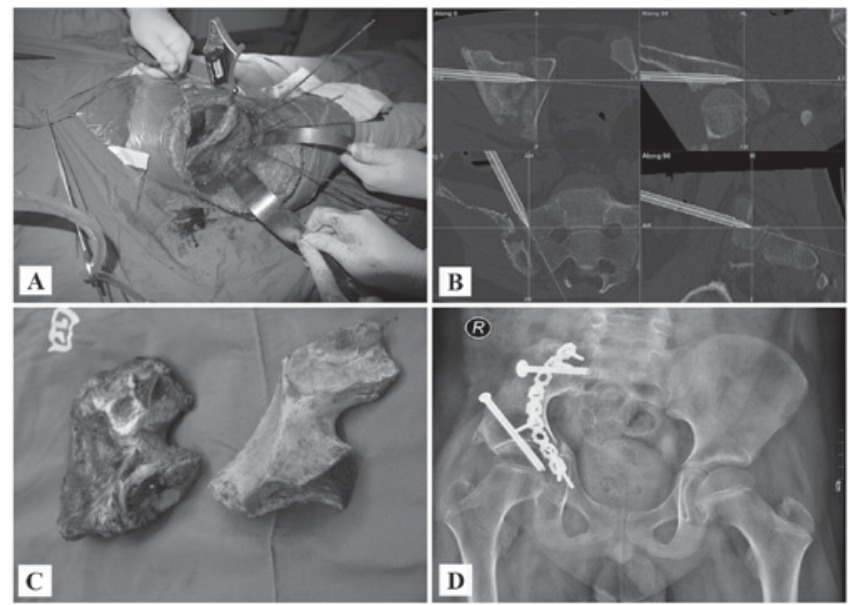

Figure 5. Type I+IIA lesion was excised and reconstructed with a plate/allograft using CANS. (A) Location of the cutting plane using Kirschner wire by CANS. (B) Intraoperative confirmation the cutting plane (screenshot of CANS). (C) After cutting and trimming, the allograft (right) matched the size of the excised tumorous tissue (left). (D) X-ray showing reconstruction at 1 year after surgery. CANS, computer-assisted navigation system.
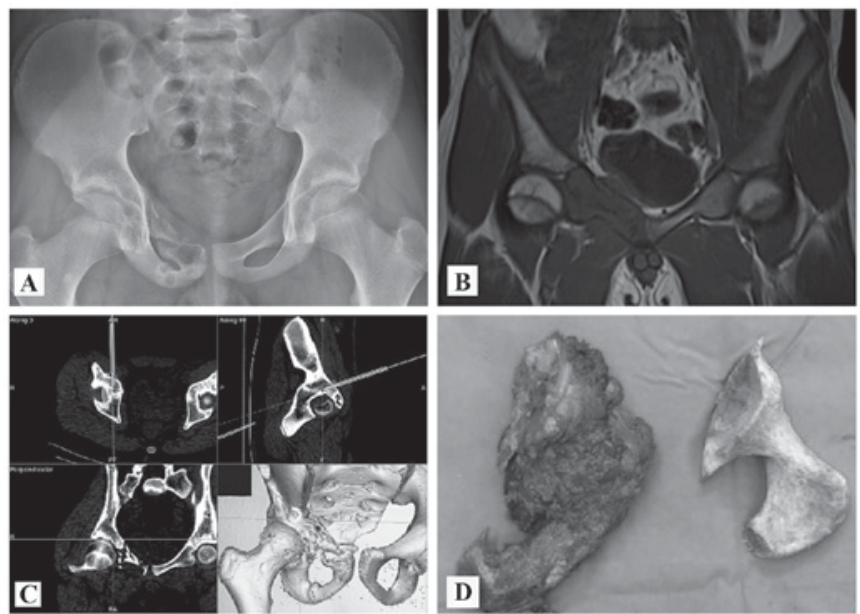

Figure 6. Type IIB+III lesion (case 10). (A) X-ray showing a large osteolytic lesion involving the anterior inferior component of the acetabulum and neighboring pubic ramus. (B) Magnetic resonance imaging indicates that the open triradiate cartilage was not penetrated by the tumor. (C) The computer-assisted navigation system was used to confirm the cutting plan during surgery. (D) The allograft (right) matched the size of excised tumor (left).

\section{Results}

Response to chemotherapy. The patients' responses to neoadjuvant chemotherapy were assessed by CT/MRI scan and graded according to RECIST. The results showed that 1 patient achieved a complete response, 9 achieved a partial response and 2 achieved stable disease (SD). No patient exhibited progressive disease. In accordance with National Comprehensive Cancer Network (NCCN) guidelines (23) for ES, excision of the tumors was attempted en bloc in all patients.

The histological response was evaluated according to a grading system developed by Ferrari et al (24). This three-grade system was defined as following: A grade I response represented a tumor with macroscopically viable

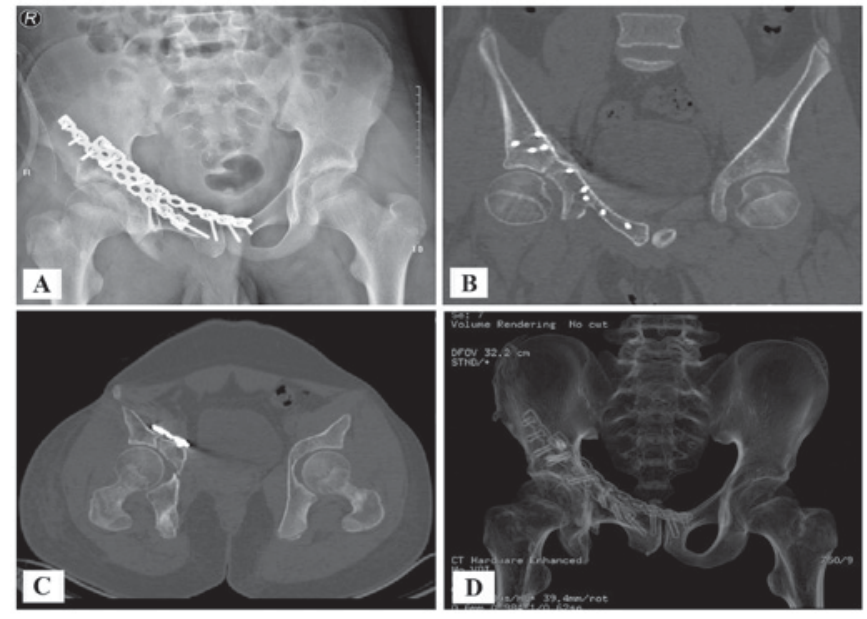

Figure 7. Type IIB+III lesion (case 10). (A) X-ray showing the reconstruction with the allograft and plate. (B and C) Computed tomography scans and (D) 3-dimensional reconstruction showing the structures of the reconstructed acetabulum following surgery.
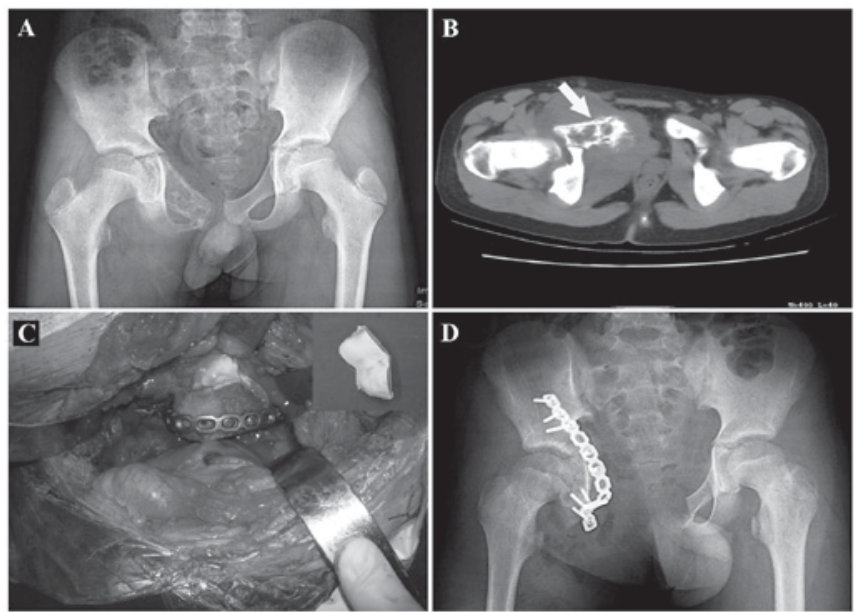

Figure 8. Type IIB+IIC+III lesion (case 7). (A) X-ray and (B) Computed tomography scan showing a large osteolytic lesion involving the whole inferior component of the acetabulum and neighboring pubis and ischium (indicated by white arrow). (C) Following tumor excision, an allograft was implanted to repair the defect. Inset of (C) shows the allograft prior to cutting and trimming. (D) X-ray showing reconstruction following surgery.

tumor areas; a grade II response represented a tumor with only microscopic foci of viable tumor; and a grade III response indicated that there was no evidence of viable tumor. In the present study, 1 patient was recorded as exhibiting a grade III response (no viable tumor cells), 8 as exhibiting a grade II response (microscopic foci) and 3 as exhibiting a grade I response (macroscopic foci).

Survival. In total, 9 patients exhibited no evidence of disease at a mean of 43 months after surgery (range, 24-72 months), 1 patient succumbed to disease at 24 months, and 2 patients remained alive with disease but had slow progressive pulmonary metastasis at 24 and 36 months, respectively. At the time of the final follow-up appointment, 11 patients remained alive (Table I).

Local recurrence. The 5 patients who received precise excision using CANS exhibited no local disease recurrence. 


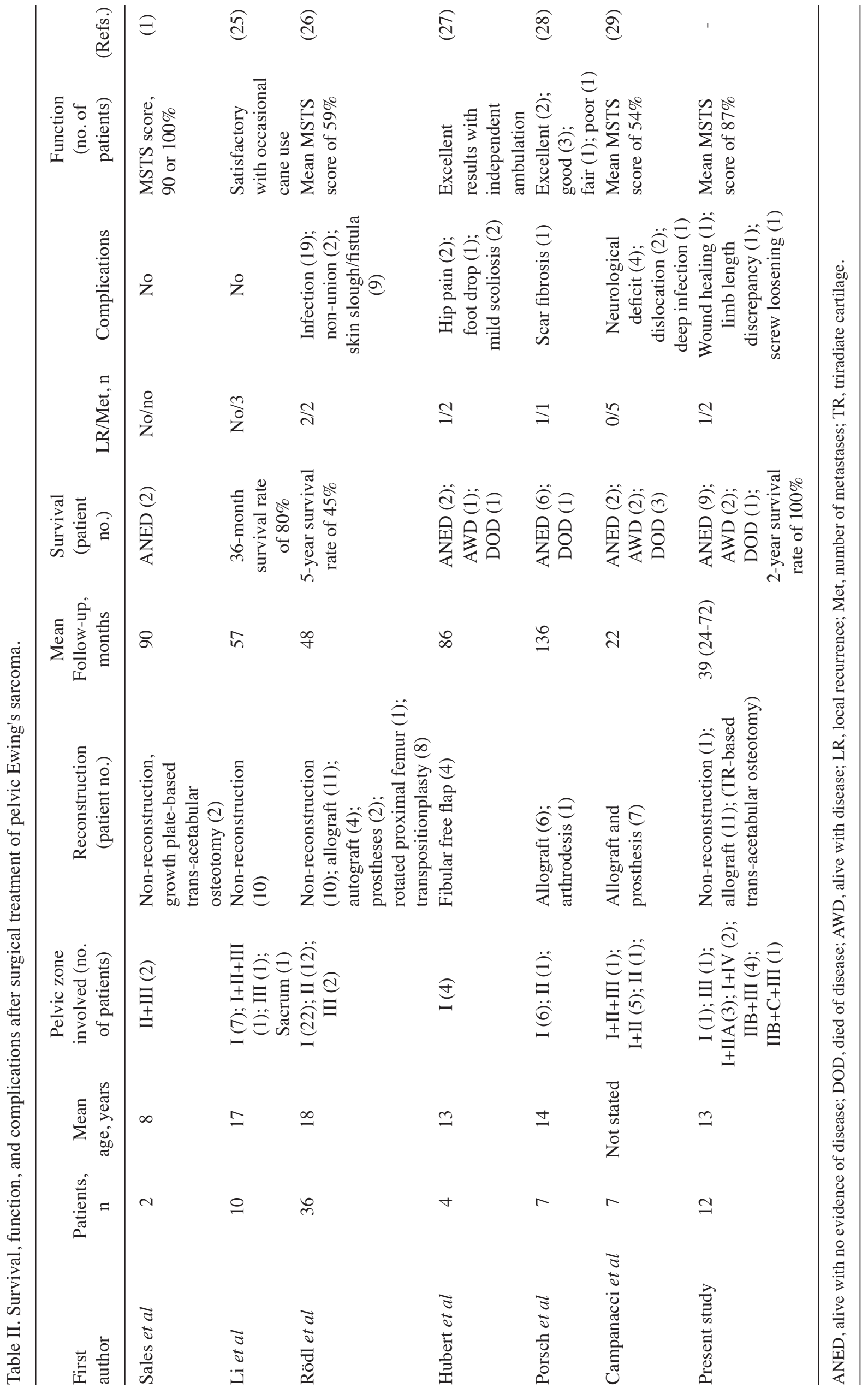



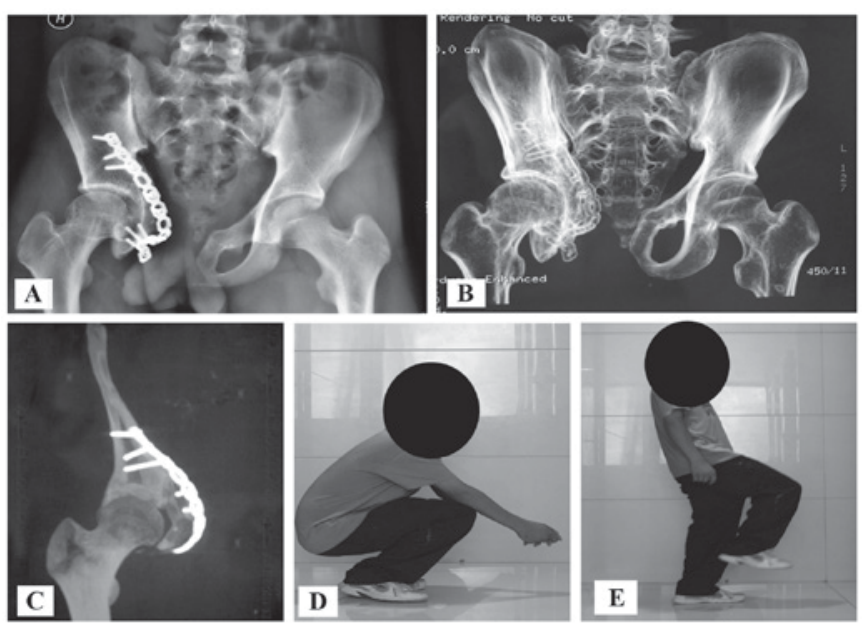

Figure 9. Type IIB+IIC+III lesion (case 7) at 3 years after surgery. (A) X-ray showing the reconstruction with allograft and plate. (B) Computed tomography scan and (C) 3-dimensional reconstruction showing the congruency of the reconstructed acetabulum. (D and E) The patient acquired good hip function following allograft transplantation.

In the 7 patients who underwent conventional resections, 1 experienced local recurrence. However, patient numbers are too small to suggest that CANS provides superior local control. The patient with local recurrence received a type I+IV resection in the first surgery. The response to neoadjuvant chemotherapy was recorded as SD according to RECIST and as grade I in Ferrari's three-grade system. At 12 months after surgery, a soft-tissue mass was found. The biopsy indicated recurrence and the patient underwent surgical intervention. This patient also had concomitant pulmonary metastasis and was alive with disease at the time of the final follow-up appointment (Table I).

Metastasis. Distant metastases developed in 3/12 patients, including 1 patient with concomitant local recurrence, at 12 , 14 and 36 months after surgery, respectively. Of the patients, 1 succumbed to the disease 10 months after metastasis was detected. The remaining 2 patients were alive with pulmonary metastases at the time of the final follow-up appointment (Table I).

Function. At the final follow-up, the patients had a mean functional score of 26 points (range, $18-28$ points). The mean radiographic score was $90.1 \%$ (range, 76-94\%), which represented an excellent radiographic result (Table I).

Complications. No patients experienced hip dislocation following acetabular reconstruction. In total, 3 patients experienced surgical complications: 1 patient had a wound healing problem due to fat liquefaction, which was superficial and healed after surgical debridement; 1 patient had a leg-length discrepancy of $2 \mathrm{~cm}$; and 1 patient had a loosening screw, for which no intervention was required.

\section{Discussion}

The excision and reconstruction of pelvic ES in children and adolesecents is challenging, particularly for periacetabular lesions $(7,8,25,26)$. Type I and type III excisions preserve the acetabulum and allow for better function. In a previous study, the defects of 4 patients were reconstructed with fibular-free flaps after type I excisions. Functionality was excellent and all patients were able to ambulate independently (27). Another study demonstrated that movement and strength were rated as 'excellent' in 6 patients following type I excisions (28). However, when patients with type II excisions were included in studies, the MSTS score decreased to 54-59\% when using conventional osteotomy. Complication rates were also varied, ranging between 71 and $100 \%(26,29)$. By contrast, the triradiate cartilage-based trans-acetabular osteotomy in the present study maximally preserved the unaffected acetabular components. The MSTS score increased to 26 (87\%), which correlated with the results of other studies (Table II) (1).

The acetabulum develops from the growth plates of the ilium, ischium and pubis. All growth plates are centrally confluent, with thick triradiate cartilage, which is a secondary ossification center of the hip bones (30). In the skeletally immature pelvis, these three bones are separated by triradiate cartilage. The centers of ossification develop at $\sim 8$ years of age and the triradiate cartilage usually closes at 16-18 years of age. The physis has long been considered to act as a barrier, preventing the spread of tumor to the epiphysis, although this barrier is not impenetrable (31). This hypothesis was supported by experimental research that identified the protein substances within the physis that inhibited angiogenesis (32). In the current study, the prerequisite for the trans-acetabular osteotomy strategy was an open triradiate cartilage without tumor invasion.

An evaluation of the association between the tumor and the triradiate cartilage is recommended when planning the resection of a tumor that involves any part of the acetabulum. A correlation study between the histological findings and MRI results in limb malignancies showed that the accuracy of MRI was $90.3 \%$ (33). The epiphysis could be safely preserved in conditions in which the tumor did not contact or only partially contacted the growth plate. The contraindication was that the growth plate was penetrated or wholly affected by the tumor (31). As the shape of the acetabulum was quite different from that of a long bone, the sagittal and coronal images captured by MRI were meticulously examined in the 8 patients with periacetabular lesions in the present study. The non-involvement of triradiate cartilage was observed in 4 patients and partial involvement was observed in another 4 . The triradiate cartilage was not penetrated or wholly affected by the tumor. After 2008, 5 patients received CANS-assisted surgeries. The reconstructed 3D tumor model allowed for the clear delineation of tumor and triradiate cartilage.

The majority of allografts in the bone bank were over-sized and required cutting or trimming to fit the defects, owing to the limited availability of pediatric allografts (Figs. 5C and 6D). This work was performed with the aid of CANS after 2008. A model of the excised bone was printed using a rapid prototyping technique. This model acted as a template for the cutting and trimming of the allograft. A CT scan revealed that the hip joint was congruent after acetabular reconstruction (Fig. 7). All of the aforementioned factors contributed to a good functional score (Tables I and II). All patients 
with lower acetabular involvement (types IIB, and IIB+IIC) received allograft reconstructions in the present study. This could benefit the growth and centralization of the femoral head. Plain radiographs indicated that the femoral head was well centered and covered at 3 years after surgery (Fig. 9). For patients with upper acetabular involvement (type IIA), the physicians strongly suggested reconstruction, as this area could bear the majority of the load in the hip joint movement. The implanted allograft did not grow like the other components of the acetabulum. To match the host's femoral head, the surgeons usually implanted a slightly larger osteoarticular allograft, which provided more space for the growth of the femoral head. Patients undergoing type I excision also required reconstruction to avoid pelvic instability and leg length discrepancy (27), whereas those undergoing type III excisions did not.

The present study had several limitations. Firstly, the study used a small number of patients. As only the children and adolescents with pelvic ES were included, it was difficult to obtain a large number of patients from one institution. Although the results cannot thoroughly prove the concept of trans-acetabular osteotomy, the data support the rationale of this technique. Secondly, this was a retrospective study with potentially uncontrolled variables, including different locations and variability in the required bone and soft tissue excisions. Finally, as only 5 patients received navigation-guided osteotomies after 2008, the present study does not have adequate power to evaluate the beneficial effects of CANS-assisted surgery. In spite of these drawbacks, the current study contributes further information on surgical strategy to treat pelvic ES.

In conclusion, this study investigated a novel surgical strategy for pelvic ES excisions in children and adolescents. Navigation was used to perform osteotomy and allograft trimming, which enhanced the accuracy of resection and reconstruction. Although it remains a small series with limited conclusions, the present study does introduce a notable surgical strategy and aids the understanding of the management of patients with rare diseases.

\section{References}

1. Sales de Gauzy J, Lafontan V, Urseï M and Accadbled F: Ewing sarcoma of the acetabulum in children: A 'growth plate-based' surgical strategy. J Pediatr Orthop 34: 326-330, 2014.

2. Devaney K, Abbondanzo SL, Shekitka KM, Wolov RB and Sweet DE: MIC2 detection in tumors of bone and adjacent soft tissues. Clin Orthop Relat Res 176-187, 1995.

3. Abed R and Grimer R: Surgical modalities in the treatment of bone sarcoma in children. Cancer Treat Rev 36: 342-347, 2010.

4. Frassica FJ, Frassica DA, Pritchard DJ, Schomberg PJ, Wold LE and Sim FH: Ewing sarcoma of the pelvis. Clinicopathological features and treatment. J Bone Joint Surg Am 75: 1457-1465, 1993.

5. Ginsberg JP, Woo SY and Johnson ME: Ewing's sarcoma family of tumors: Ewing's sarcoma of bone and soft tissue and the peripheral primitive neuroectodermal tumors, in Pizzo PA, Poplack DG (eds): Principles and Practice of Pediatric Oncology. 4th edition. Philidelphia, PA, Lippincott Williams \& Wilkins, pp973-101614, 2002.

6. Falk S and Alpert M. Five year survival of patients with Ewing's sarcoma. Surg Gynecol Obstet 124: 319-324, 1967.

7. Bacci G, Ferrari S, Bertoni F, Rimondini S, Longhi A, Bacchini P, Forni C, Manfrini M, Donati D and Picci P: Prognostic factors in nonmetastatic Ewing's sarcoma of bone treated with adjuvant chemotherapy: Analysis of 359 patients at the Istituto Rizzoli. J Clin Oncol 18: 4-11, 2000.
8. Bhagat S, Sharma H, Pillai DS and Jane MJ: Pelvic Ewing's sarcoma: A review from Scottish Bone Tumour Registry. J Orthop Surg (Hong Kong) 16: 333-338, 2008.

9. Sucato DJ, Rougraff B, McGrath BE, Sizinski J, Davis M, Papandonatos G, Green D, Szarzanowicz T and Mindell ER: Ewing's sarcoma of the pelvis. Long-term survival and functional outcome. Clin Orthop Relat Res 193-201, 2000.

10. Enneking WF and Dunham WK: Resection and reconstruction for primary neo-plasms involving the innominate bone. J Bone Joint Surg Am 60: 731-746, 1978.

11. O'Connor MI and Sim FH: Salvage of the limb in the treatment of malignant pelvic tumors. J Bone Joint Surg Am 71: 481-494, 1989.

12. Jansen JA, van de Sande MA and Dijkstra PD: Poor long-term clinical results of saddle prosthesis after resection of periacetabular tumors. Clin Orthop Relat Res 471: 324-331, 2013.

13. Gebert C, Wessling M, Hoffmann C, Roedl R, Winkelmann W, Gosheger G and Hardes J: Hip transposition as a limb salvage procedure following the resection of periacetabular tumors. J Surg Oncol 103: 269-275, 2011.

14. Liporace FA, Ong B, Mohaideen A, Ong A and Koval KJ. Development and injury of the triradiate cartilage with its effects on acetabular development: Review of the literature. J Trauma 54: 1245-1249, 2003.

15. Ponseti IV: Growth and development of the acetabulum in the normal child. Anatomical, histological, and roentgenographic studies. J Bone Joint Surg Am 60: 575-585, 1978.

16. Cheung WH, Lee KM, Fung KP and Leung KS: Growth plate chondrocytes inhibit neo-angiogenesis: A possible mechanism for tumor control. Cancer Lett 163: 25-32, 2001.

17. Winkelmann W: A new surgical method in malignant tumors of the ilium. Z Orthop Ihre Grenzgeb 126: 671-674, 1988 (In German).

18. Therasse P, Arbuck SG, Eisenhauer EA, Wanders J, Kaplan RS, Rubinstein L, Verweij J, Van Glabbeke M, van Oosterom AT, Christian MC and Gwyther SG: New Guidelines to Evaluate the Response to Treatment in Solid Tumors. J Natl Cancer Inst 92: 205-216, 2000.

19. Enneking WF, Spanier SS and Goodman MA: A system for the surgical staging of musculoskeletal sarcoma. Clin Orthop Relat Res 106-120, 1980.

20. Fan H, Guo Z, Wang Z, Li J and Li X. Surgical technique: Unicondylar osteoallograft prosthesis composite in tumor limb salvage surgery. Clin Orthop Relat Res 470: 3577-3586, 2012.

21. Enneking WF, Dunham W, Gebhardt MC, Malawar M and Pritchard DJ: A system for the functional evaluation of reconstructive procedures after surgical treatment of tumors of the musculoskeletal system. Clin Orthop Relat Res 241-246, 1993.

22. Glasser D and Langlais F: The ISOLS radiological implant evaluation system. In: Langlais F and Tomeno B (eds). Limb Salvage: Major Reconstructions in Oncologic and Nontumoral Conditions. Springer-Verlag, Heidelberg, Germany, pp23-31, 1991.

23. Biermann JS, Chow W, Reed DR, Lucas D, Adkins DR, Agulnik M, Benjamin RS, Brigman B, Budd GT, Curry WT, et al: NCCN Guidelines Insights: Bone Cancer, Version 2.2017. J Natl Compr Canc Netw 15: 155-167, 2017.

24. Ferrari S, Bertoni F, Palmerini E, Errani C, Bacchini P, Pignotti E, Mercuri M, Longhi A, Cesari M and Picci P: Predictive factors of histologic response to primary chemotherapy in patients with Ewing sarcoma. J Pediatr Hematol Oncol 29: 364-368, 2007.

25. Li WK, Lane JM, Rosen G, Marcove RC, Caparros B, Huvos A and Groshen S. Pelvic Ewing's sarcoma. Advances in treatment. J Bone Joint Surg Am 65: 738-747, 1983.

26. Rödl RW, Hoffmann C, Gosheger G, Leidinger B, Jürgens $H$ and Winkelmann W: Ewing's sarcoma of the pelvis: Combined surgery and radiotherapy treatment. J Surg Oncol 83: 154-160, 2003.

27. Hubert DM, Low DW, Serletti JM, Chang B and Dormans JP: Fibula free flap reconstruction of the pelvis in children after limb-sparing internal hemipelvectomy for bone sarcoma. Plast Reconstr Surg 125: 195-200, 2010.

28. Porsch M, Kornhuber B and Hovy L: Functional results after partial pelvic resection in Ewing's sarcoma of the ilium. Arch Orthop Trauma Surg 119: 199-204, 1999. 
29. Campanacci D, Chacon S, Mondanelli N, Beltrami G, Scoccianti G, Caff G, Frenos F and Capanna R: Pelvic massive allograft reconstruction after bone tumour resection. Int Orthop 36: 2529-2536, 2012.

30. Portinaro NM, Murray DW and Benson MK: Microanatomy of the acetabular cavity and its relation to growth. J Bone Joint Surg Br 83: 377-383, 2001.

31. Panuel M, Gentet JC, Scheiner C, Jouve JL, Bollini G, Petit P, Bourliere-Najean B and Devred P: Physeal and epiphyseal extent of primary malignant bone tumors in childhood. Correlation of preoperative MRI and the pathologic examination. Pediatr Radiol 23: 421-424, 1993.
32. Langer R, Brem H, Falterman K, Klein M and Folkman J: Isolations of a cartilage factor that inhibits tumor neovascularization. Science 193: 70-72, 1976.

33. San-Julian M, Aquerreta JD, Benito A and Cañadell J: Indications for epiphyseal preservation in metaphyseal malignant bone tumors of children: Relationship between image methods and histological findings. J Pediatr Orthop 19: 543-548, 1999. 